\title{
ANÁLISE DO PADRÃO DE SEXO E FAIXA ETÁRIA DOS FALECIDOS DE CÂNCER DE ESÔFAGO NO SUL DO BRASIL
}

\section{ANALYSIS OF SEX AND AGE GROUP PATHER OF THE DEAD BY ESOPHAGUS CANCER IN SOUTH OF BRAZIL}

\author{
Gabriel Rodrigues Rocha e Silva \\ Universidade de Brasília, Departamento de Geografia, Brasília, DF, Brasil \\ gabrielrodrigues123457@gmail.com \\ Helen Gurgel \\ Universidade de Brasília, Departamento de Geografia, Brasília, DF, Brasil \\ helengurgel@unb.br \\ Luiz Belino Ferreira Sales \\ Mestre em Desenvolvimento e Meio Ambiente pela Universidade Federal do Ceará \\ luizbelino@gmail.com
}

\begin{abstract}
RESUMO
A pesquisa tem por objetivo descrever a dinâmica espaço-temporal dos óbitos por câncer de esôfago na região Sul do Brasil, a partir de sexo e faixa etária, durante os anos de 2007 a 2017, e segundo municípios de residência. A pesquisa trata-se de um estudo descritivo, de abordagem metodológica quantitativa. A distribuição temporal anual de casos é contínua e a distribuição espacial de taxas de mortalidade é concentrada nas mesorregiões do Noroeste Rio-Grandense, Região Metropolitana de Porto Alegre e Centro Oriental Rio-Grandense para ambos os sexos. A maior mortalidade é masculina, seguindo a tendência mundial e brasileira. Se concentram na faixa etária dos 75-79 anos os óbitos femininos e aos 60-64 anos os masculinos no Rio Grande do Sul. No Paraná e em Santa Catarina, o ápice de óbitos de mulheres é na faixa dos 65-69 anos e o dos homens é aos 60-64 anos. Esta pesquisa atualiza o perfil epidemiológico da região.
\end{abstract}

Palavras-chave: Câncer de esôfago. Região Sul. Taxa de mortalidade. Sexo. Faixa etária.

\begin{abstract}
The research aims to describe the temporal-spatial dynamics of deaths from esophageal cancer in the southern region of Brazil, from sex and age group, during the years 2007 to 2017 , according to municipalities of residence. The research is a descriptive study, with a quantitative methodological approach. The annual temporal distribution of cases is continuous and the spatial distribution of mortality rates is concentrated in the mesoregions of the Northwest Rio-Grandense, Metropolitan Region of Porto Alegre and Centro Oriental RioGrandense for both sexes. Due to the higher male mortality, following the world and Brazilian trend, the mesoregions of the Northeast Rio-Grandense, Southwest Paranaense, North Central Paranaense and Western Catarinense present concentrations for this sex. Female deaths are concentrated in the 75-79 year age group and in the male deaths in the 60-64 year age group in Rio Grande do Sul. In Paraná and Santa Catarina, the peak of women's deaths is in the 65-69 age group and the of men is at 60-64 years old. This research updates the epidemiological profile of the region, which, when mentioned, did not go beyond the regional analysis, so it also deepens it.
\end{abstract}

Keywords: Esophageal cancer. South Region. Mortality rate. Sex. Age group.

Recebido em: 15/01/2022

Aceito para publicação em: 27/01/2022.

$\begin{array}{lllll}\text { Hygeia } & \text { Uberlândia - MG } \quad \text { Edição especial: X GeoSaude } & \text { Fev./2022 } & \text { p. 155-167 } & \text { Página } 155\end{array}$




\section{INTRODUÇÃO}

De acordo com o Relatório Mundial do Câncer de 2020, produzido pela Agência Internacional de Pesquisa em Câncer (IARC, sigla em inglês), agência especializada da Organização Mundial da Saúde (OMS) em neoplasias, o câncer de esôfago foi o oitavo tipo de neoplasia maligna mais recorrente mundialmente e ocupa o sexto lugar como principal causador de óbitos ao redor do mundo (WHO, 2020).

O câncer se demonstra como um desafio notável para os países classificados como desenvolvidos e em desenvolvimento que estão em estágio de menor demanda por atendimento a doenças infecto parasitárias, pois passaram ou estão passando por uma transição epidemiológica (GUERRA, GALLO, MENDONÇA, 2005; WHO, 2020). Entendemos que essa mudança aponta para a necessidade de volver gradativamente a atenção para o planejamento/estabelecimento de uma assistência em saúde capaz de lidar com doenças crônico-degenerativas e suas ramificações. No entanto, em casos de uma transição epidemiológica incompleta, cenário caracterizado por Carmo (2020) como um fenômeno que consiste em uma redução relativa de óbitos por doenças transmissíveis em um cenário no qual as mesmas coexistem com o aumento de doenças crônico-degenerativas, o sistema de saúde deve estar preparado para lidar com ambas realidades concomitante. Esse é o caso de países na América Latina, com tendências epidemiológicas que apresentam padrões de comportamento distinto do modelo inicial de transição epidemiológica (CARMO, 2020).

No Brasil, segundo análise exploratória do Sistema de Informação sobre Mortalidade (SIM), nos anos de 2007 e 2008, o câncer de esôfago foi a sexta causa de morte mais frequente por neoplasia maligna no país, evoluindo para a sétima posição em 2009 e, da mesma forma, em 2010. A partir de 2011 e até 2017, o câncer de esôfago permaneceu como a oitava maior mortalidade dentre as enfermidades do Capítulo 2 - Neoplasia [tumores] - da atual Classificação Estatística Internacional de Doenças e Problemas Relacionados à Saúde (CID-10), colocação compatível com a atual posição dessa neoplasia mundialmente. Segundo o Instituto Nacional do Câncer (INCA, 2020), estima-se que em 2020 tenham 11.390 novos casos de câncer de esôfago no Brasil, majoritariamente na população masculina $(76,2 \%)$.

O código C15 da CID-10 corresponde ao câncer de esôfago, agrupando o carcinoma das células escamosas do esôfago (CEE) e o adenocarcinoma de esôfago, ambos com fatores de risco e perfil epidemiológico específicos (WHO, 2020).

Ainda de acordo com o Sistema de Informações sobre Mortalidade, o Rio Grande do Sul liderou, em comparação às demais unidades da federação, no quesito mortalidade por câncer de esôfago entre os anos de 2007 a 2017. Os estados do Paraná e Santa Catarina sempre estiveram entre as cinco maiores taxas de mortalidade por neoplasias malignas de esôfago. Segundo o mesmo banco de dados, os estados da região Sul congregaram 21.518 óbitos nesse período por conta da neoplasia em questão, sendo o motivo do recorte espacial focado na Região Sul.

A presente pesquisa tem como objetivo descrever a dinâmica espaço-temporal dos óbitos por câncer de esôfago na região Sul do Brasil, a partir de sexo e faixa etária, durante os anos de 2007 a 2017 e segundo municípios de residência. A caracterização foi realizada com as colunas de idade e sexo biológico do Sistema de Informação sobre Mortalidade (SIM).

\section{Fatores de risco do câncer de esôfago}

Os principais fatores de risco conhecidos atualmente para o câncer esofágico no mundo são o álcool e o tabaco (WHO, 2014; WHO, 2020). Entretanto, o consumo exagerado de gorduras, a baixa ingestão de fibra, o sedentarismo e o consumo de bebidas quentes demais, quando excede a marca de 65으, são considerados agentes carcinogênicos (BAÚ, HUTH, 2011, WHO, 2014). Não há estudo abrangente o suficiente que indique uma predisposição genética para o câncer de esôfago, mas há estudos que apontam a possibilidade de associação (WHO, 2020).

Atualmente, a Lista de Classificação da Organização Mundial da Saúde explicita sete agentes carcinogênicos com evidência suficiente para confirmar como causadores do câncer de esôfago. Sendo eles, acetaldeído associado com o consumo de bebidas alcoólicas, betel quid com tabaco e sem tabaco, tabaco com e sem fumaça, radiação X e gama. Na classe de agentes carcinogênicos com evidências limitadas, ou seja, que tem estudos que apontam a relação, porém necessitam de mais estudos que embasam a relação causal, estão a lavagem a seco para os profissionais da área, picles tradicional asiático, indústria de produção de borracha e o consumo de bebidas muito quente (WHO, 2019). 
Os fatores de risco dos dois tipos de câncer de esôfago, apresentados na Quadro 1, foram divulgados pelo Relatório Mundial do Câncer de 2020 e apresentam níveis de associação positiva cientificamente embasada.

Quadro 1 - Associação positiva do Fatores de Risco, segundo a revisão da literatura científica.

\begin{tabular}{|l|l|l|}
\hline Fatores de Risco & $\begin{array}{l}\text { Carcinoma escamoso de } \\
\text { esôfago }\end{array}$ & $\begin{array}{l}\text { Adenocarcinoma de } \\
\text { esôfago }\end{array}$ \\
\hline Sexo Biológico & $\begin{array}{l}\text { Maior disposição em } \\
\text { Homens do que em Mulheres }\end{array}$ & $\begin{array}{l}\text { Maior disposição em } \\
\text { Homens do que em Mulheres }\end{array}$ \\
\hline Raça & $\begin{array}{l}\text { Maior disposição em Negros } \\
\text { do que em Brancos }\end{array}$ & $\begin{array}{l}\text { Maior disposição em } \\
\text { Brancos do que em Negros }\end{array}$ \\
\hline Obesidade & Dados limitados & Muitas associações \\
\hline Uso de Tabaco & Muitas associações & Poucas associações \\
\hline Consumo de Álcool & Muitas associações & Sem associação \\
\hline $\begin{array}{l}\text { Doença do refluxo } \\
\text { gastresofágico }\end{array}$ & Sem dados & Muitas associações \\
\hline Suscetibilidade Genética & Poucas associações & Pouquíssimas associações \\
\hline Bebidas muito quentes & Algumas associações & Sem dados \\
\hline Baixo status socioeconômico & Algumas associações & Dados limitados \\
\hline $\begin{array}{l}\text { Infecção por Helicobacter } \\
\text { pylori }\end{array}$ & Sem associação & Associação protetiva \\
\hline Má higiene oral & Poucas associações & Dados limitados \\
\hline $\begin{array}{l}\text { Uso de Ópio } \\
\text { Poluição do ar em ambientes } \\
\text { internos }\end{array}$ & Poucas associações & Sem dados \\
\hline Água não encanada & Pouquíssimas associações & Sem dados \\
\hline
\end{tabular}

Fonte: Relatório Mundial do Câncer (WHO, 2020, p. 327). Adaptado pelos autores.

Consoante a IARC (WHO, 2020), a inalação de queima de material orgânico e mineral em ambientes de baixa ventilação pode ocasionar regiões de alta incidência de CEE, assim como a combustão em automóveis e a queima de combustíveis industriais.

Em áreas de cluster em que a proporção de gênero é equilibrada, diferentes fatores, além do álcool e tabaco, podem estar associados. Para citar alguns exemplos, o déficit de vitaminas, especialmente do composto orgânico riboflavina, a ausência de água encanada, a baixa ingestão de selênio, a má higiene bucal e/ou uso de ópio são agravantes. Em ambientes ocupacionais, as indústrias têxtil e de fundição de metal são classificadas como fatores de risco ambiental aos seus trabalhadores (WHO, 2020).

Em nível nacional, o documento "Diretrizes Diagnósticas e Terapêuticas para o carcinoma esofágico do Brasil" considera o fumo, a obesidade e o consumo de álcool como os principais fatores de risco. Outros fatores de risco menos frequentes, segundo a Portaria, são "a tilose ou Síndrome PlummerVinso [...], anemia ferropriva e membranas esofágicas; e acalasia [...]" (BRASIL, 2014, p. 2).

Em relação ao adenocarcinoma, o esôfago de Barret e a obesidade consistem nos fatores de risco preponderantes. Estudos observacionais sobre o efeito protetor de medicamentos, como aspirina e anti-inflamatórios não esteroidais, e dieta rica em fibra foram reportados positivamente (BRASIL, 2014).

\section{A bebida quente no Sul}

No caso do carcinoma escamoso de esôfago, as bebidas muito quentes compõem o Grupo $2 \mathrm{~A}$ (Provavelmente Cancerígeno), sendo uma questão para diversas culturas que têm o hábito de consumir bebidas locais em temperatura quente. Estudos realizados na Tanzânia (MUNISHI, et al., 2015), Irã

$\begin{array}{lllll}\text { Hygeia Uberlândia - MG } & \text { Edição especial:X GeoSaude } & \text { Fev./2022 } & \text { p. 155-167 } & \text { Página } 157\end{array}$ 
(MUÑOZ, et al., 1982; ISLAMI, et al., 2009), China (MUÑOZ, et al., 1982; LIN; et al, 2011), Turquia (KOCA, et al., 2018), Singapura (JONG, et al., 1974), França (LAUNOY, et al., 1998), América do Sul (CASTELLSAGUÉ, et al., 2000), Uruguai (STEFANI, et al., 1990) e, inclusive, Sul do Brasil (VICTORA, et al., 1987) sustentam a preocupação em relação ao consumo de diversas bebidas em temperaturas excessivamente elevadas.

O chimarrão é uma bebida produzida pela erva llex paraguariensis, nativa do Sul do Brasil, e é reconhecido como parte do alicerce de construção da identidade histórica, cultural e econômica do país (OLIVEIRA; ROTTA, 1983; ASSMANN, 2018). No Sul do Brasil, o chimarrão causa maior preocupação do que o café, apesar de ambas serem mencionadas pela OMS, por ser uma bebida regional, consumida próximo a $70^{\circ} \mathrm{C}$ e ingerida com um canudo, chamado bomba, que leva a bebida para a parte posterior da língua e orofaringe resultando, gradativamente, em lesões (BARROS, et al., 2000; WHO, 2016; ASSMANN, 2018).

\section{DADOS E METODOLOGIA}

A pesquisa trata-se de um estudo descritivo do padrão de sexo e faixa etária dos falecidos por neoplasia maligna do esôfago no Rio Grande do Sul, Santa Catarina e Paraná, entre os anos de 2007 a 2017, isto é, de janeiro de 2007 a dezembro de 2017.

Os dados disponibilizados pelo Departamento de Informática do Sistema Único de Saúde (DATASUS) foram obtidos em formato DBC. Os quais, foram convertidos em XLS em software livre para análise de dados, chamado RStudio, permitindo analisar os dados em editor de planilha eletrônica. Foi utilizado para tal o Microsoft Excel 2013, onde foi realizada a estatística básica dos dados e gerado tabelas e gráficos que compõem essa pesquisa.

O Sistema de Informações sobre Mortalidade (SIM) foi empregado na coleta dos dados referentes aos óbitos para as Unidades da Federação do Sul do Brasil nos 11 anos, que se iniciaram em 2007. Os dados foram selecionados a partir da causa base do óbito de código C15 da Classificação Internacional de Doenças - 10ª revisão (CID-10), ou seja, o câncer de esôfago, e filtrados segundo os estados do Paraná, Santa Catarina e Rio Grande do Sul.

As faixas etárias foram separadas em intervalo de 4 anos de acordo com a coluna IDADE do SIM.

O cálculo das taxas de mortalidade por sexo foi feito com denominadores obtidos de estimativas do Ministério da Saúde, disponibilizados durante a pandemia do COVID-19, e o cálculo das taxas seguiram a seguinte equação:

Número de óbitos de homens ou mulheres residentes falecidas por C15 $\quad \times 100.000$

População total residente de homens ou mulheres, de acordo com sexo do numerador

O cálculo das taxas de mortalidade estadual seguiu a seguinte equação:

Número de óbitos de homens ou mulheres residentes falecidas por $\mathrm{C} 15$ $x 1.000 .000$

População total residente

\section{RESULTADOS E DISCUSSÃO}

A construção de um perfil epidemiológico subsidia o setor de saúde em ações de planejamento e execução de serviços, como campanhas de prevenção aos fatores de risco, focados nas pessoas com as características dos grupos mais afetados. Tendo isso em mente, a região foi selecionada para ser comparada aos perfis nacionais publicados previamente.

A região do estudo foi selecionada em análise exploratória dos falecimentos por câncer de esôfago em escala estadual, onde foi identificado que os três estados do Sul estavam sempre entre as cinco maiores taxas de mortalidade para essa enfermidade, entre 2007 e 2017 (Figura 1). Dentre as regiões, - Sudeste vem logo em seguida com as maiores taxas de mortalidade, sendo o Espírito Santo, recorrentemente, o segundo estado com a maior delas, atrás apenas do Rio Grande do Sul, o qual mantem a liderança em todo recorte temporal. 
Figura 1 - Análise exploratória da taxa de mortalidade do câncer de esôfago nas Unidades da Federação (2007-2017).
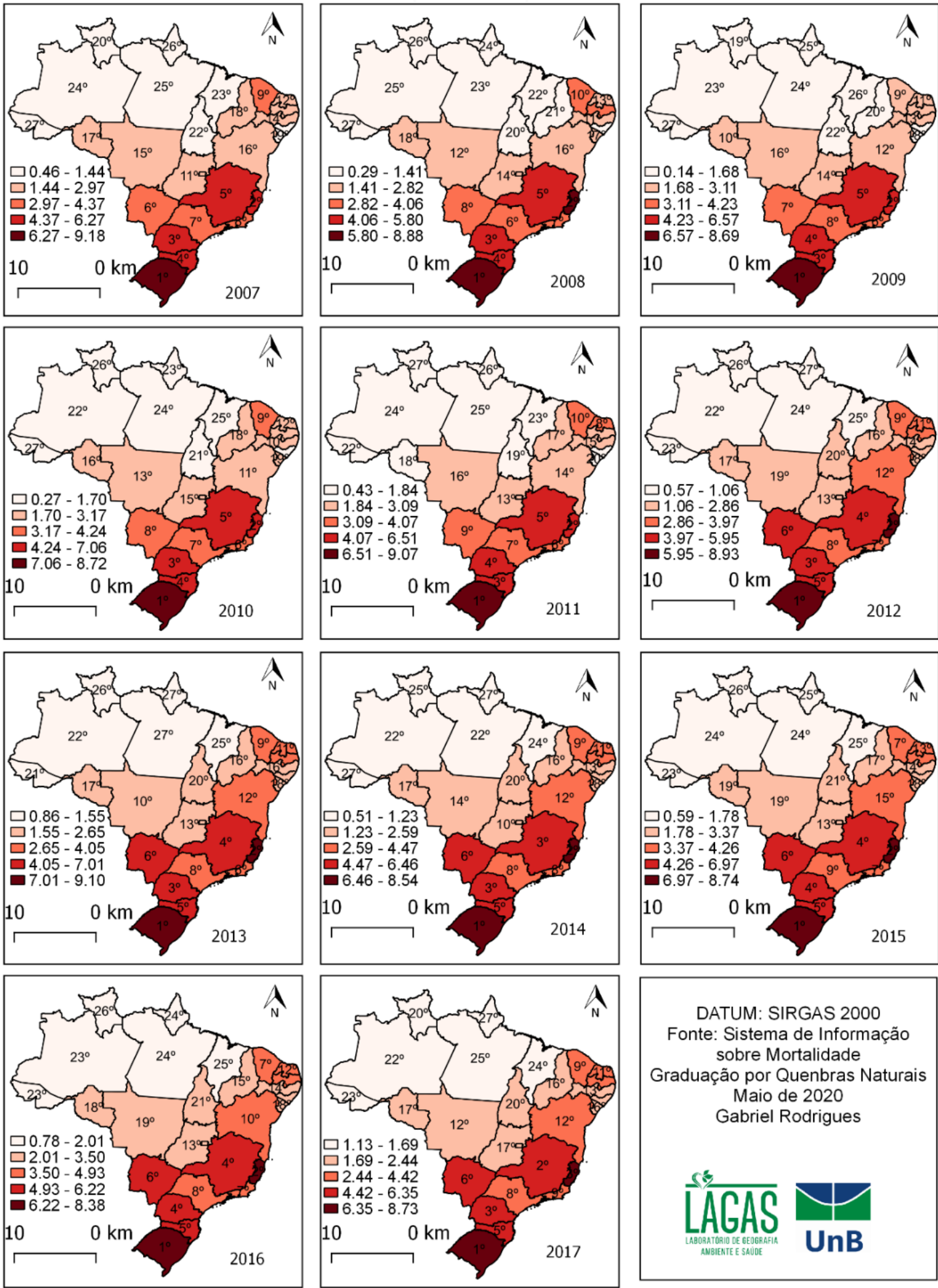

Fonte: MINISTÉRIO DA SAÚDE, 2020. Elaboração: os autores (2020).

Conforme demonstrado na Figura 2, a região Sul não apresenta um aumento anual circunstancial de óbitos, assim como nenhum dos estados quando analisados individualmente. 
Figura 2 - Análise temporal dos óbitos absolutos por câncer de esôfago na Região Sul (2007-2017).

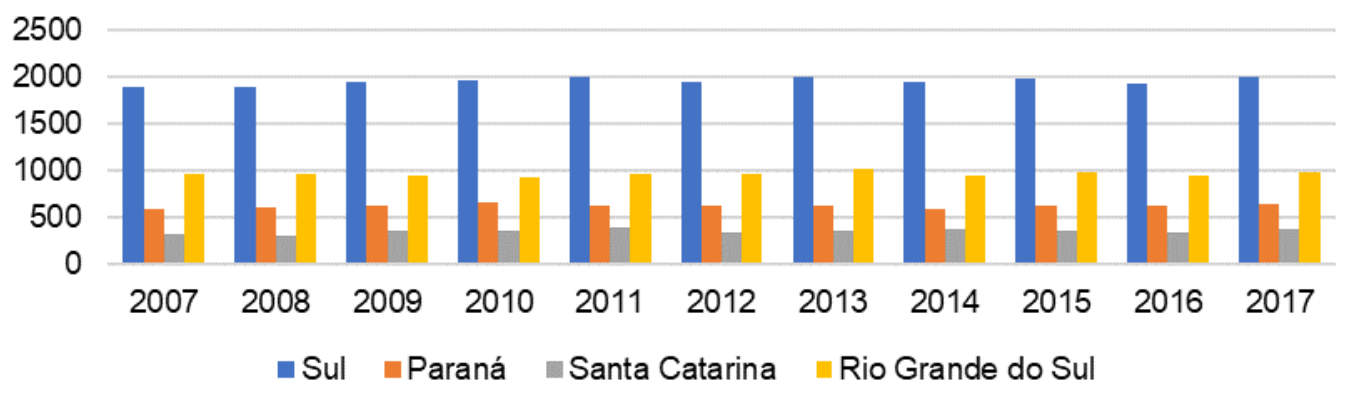

Fonte: MINISTÉRIO DA SAÚDE, 2020. Elaboração: os autores (2020).

\section{Sexo}

A organização da variável sexo biológico foi organizada segundo a linha SEXO do banco de dados do SIM. A categoria foi preenchida com Homem e Mulher para os óbitos por câncer de esôfago, com a ocorrência de um único registro da categoria "Ignorado".

Nos três estados analisados, os homens compõem a maioria da amostra, resultado compatível ao nível nacional e mundial (BRAGA; DUTRA; OLIVEIRA, 2011). Em Santa Catarina, 80,8\% $(n=3.214)$ dos falecidos eram do sexo masculino, enquanto apenas $19,2 \%(n=763)$ eram do sexo feminino, sendo este o estado de maior diferença proporcional entre os sexos. No Paraná, $77,7 \%(n=5.354)$ dos falecidos foram registrados como masculino e $22,3 \%(n=1.540)$ como feminino. $O$ estado com mais mortes na região, o Rio Grande do Sul, teve a maior proporção de mulheres que vieram à óbito pela enfermidade, representando $25,0 \%(\mathrm{n}=2.666)$ dos falecimentos, ao passo que os homens representaram $75,0 \%(n=7.977)$ dos óbitos por câncer de esôfago no estado. Em um estudo realizado com os dados de 1979 a 1989 no estado gaúcho já apontava para essa tendência dos óbitos do sexo masculino (PROLLA, DIETZ, COSTA, 1993).

Segundo o INCA (2020), o Brasil perdeu 8.554 pessoas para a doença em 2017, sendo a média nacional composta por $77,7 \%$ por homens e $22,3 \%$ por mulheres, ou seja, a porcentagem feminina em Santa Catarina está abaixo da média nacional, a do Paraná está próxima e a do Rio Grande do Sul acima.

A mesorregião do Noroeste Rio-Grandense anualmente se destaca negativamente por causa de altas taxas de mortalidade feminina por câncer de esôfago (Figura 3). Há uma certa linearidade municipal de altas taxas de mortalidade, tanto masculina (Figura 4) quanto feminina (Figura 3), partindo da Região Metropolitana de Porto Alegre em direção a mesorregião Centro Oriental Rio-Grandense, levantando hipóteses, como, por exemplo, a possibilidade da concentração de indústrias têxtil, de fundição de metal e de produção de borracha podem afetar os trabalhadores. Para a comprovação dessa hipótese é necessário analisar o perfil ocupacional dos acometidos e realizar uma análise intramunicipal do perfil produtivo. Devemos lembrar que outros fatores, como poluição e ausência de água encanada, afetam ambos os sexos (WHO, 2020), podendo também estarem envolvidos nessas distribuições espaciais de altas taxas.

A mesorregião Oeste Catarinense tem alguns municípios com altas taxas de mortalidade feminina, mas não ao ponto de criar um padrão espacial municipal anual, pois há uma variabilidade dentro da própria sub-região.

Os municípios do Paraná se destacam negativamente apenas no ano de 2013, mas nos demais anos estudados apresenta baixas taxas de mortalidade em comparação com outros municípios da região Sul.

Do fato do maior número de óbitos serem de homens, os mapas de distribuição espacial das taxas de mortalidade por câncer de esôfago em homens têm um padrão mais complexo e exibem maior número de municípios com altas taxas de mortalidade (Figura 4).

A taxa de mortalidade entre homens mostra um destaque para o norte do Rio Grande do Sul, precisamente nas mesorregiões do Noroeste Rio-Grandense, Nordeste Rio-Grandense, Centro Oriental Rio-Grandense e Região Metropolitana de Porto Alegre, com altas taxas anualmente. Enquanto isso, o sul do estado segue um padrão de baixas taxas de mortalidade de câncer de esôfago em homens. Este último resultado difere dos encontrados por Prolla, Dietz e Costa (1993) ao estudar

Hygeia Uberlândia - MG $\quad$ Edição especial: X GeoSaude $\quad$ Fev./2022 p. 155-167 Página 160


a distribuição da mortalidade do câncer de esôfago no Rio Grande do Sul de 1979 a 1989, onde apontam uma concentração nas microrregiões no sul do estado.

O Paraná tem duas mesorregiões com altas taxas de mortalidade, Sudoeste Paranaense e Norte Central Paranaense. No caso do estado de Santa Catarina, o Oeste Catarinense anualmente evidencia altas taxas de mortalidade de homens.

Figura 3 - Distribuição espaço-temporal da taxa de mortalidade de câncer de esôfago em mulheres de 2007 a 2017.
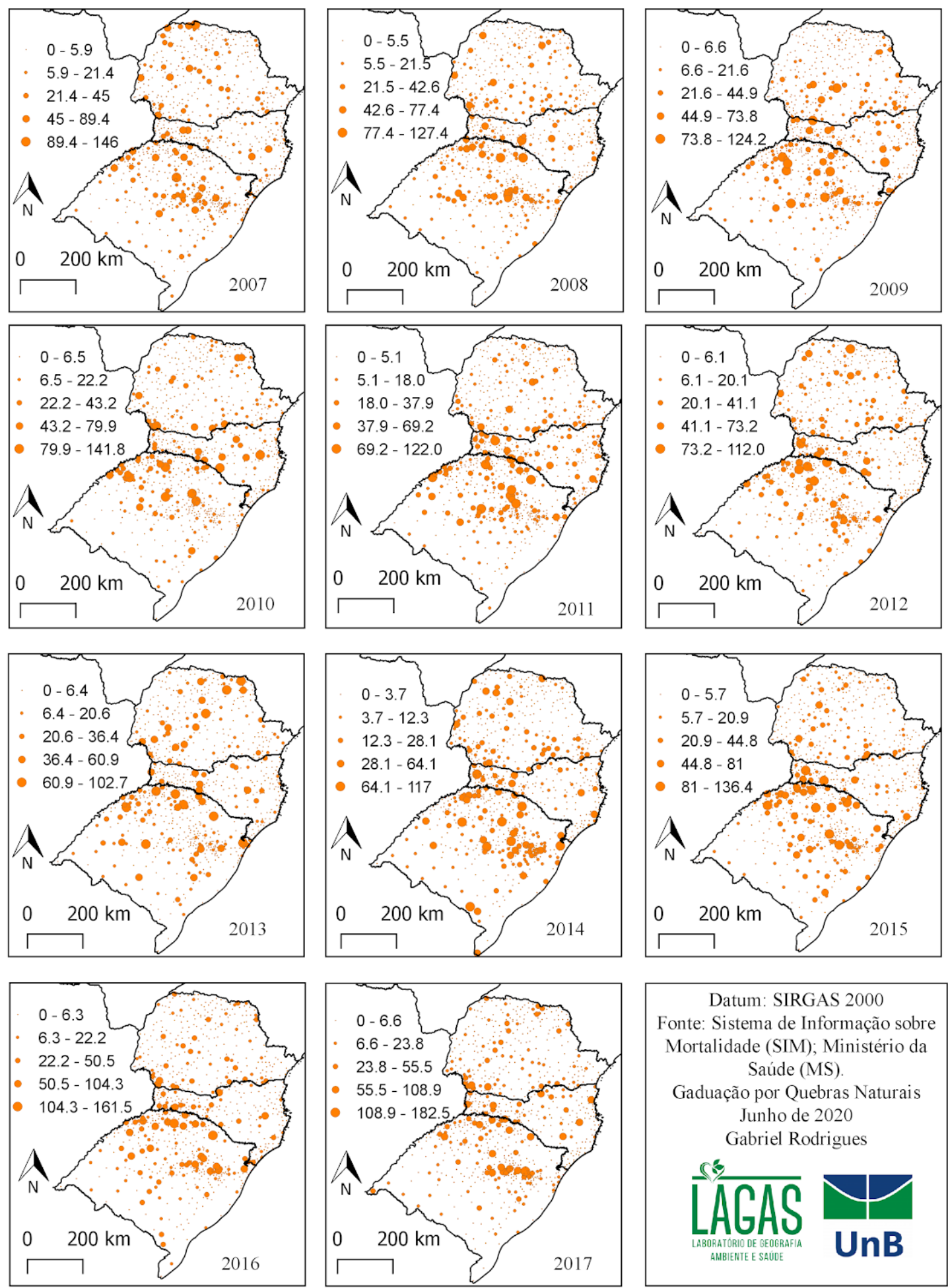

Datum: SIRGAS 2000

Fonte: Sistema de Informação sobre Mortalidade (SIM); Ministério da Saúde (MS)

Gaduação por Quebras Naturais Junho de 2020

Gabriel Rodrigues

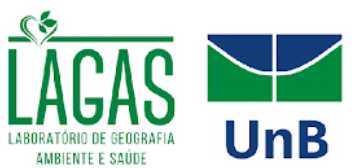

Fonte: MINISTÉRIO DA SAÚDE, 2020. Elaboração: os autores (2020). 
Figura 4 - Distribuição espaço-temporal da Taxa de mortalidade de câncer de esôfago em homens de 2007 a 2017.
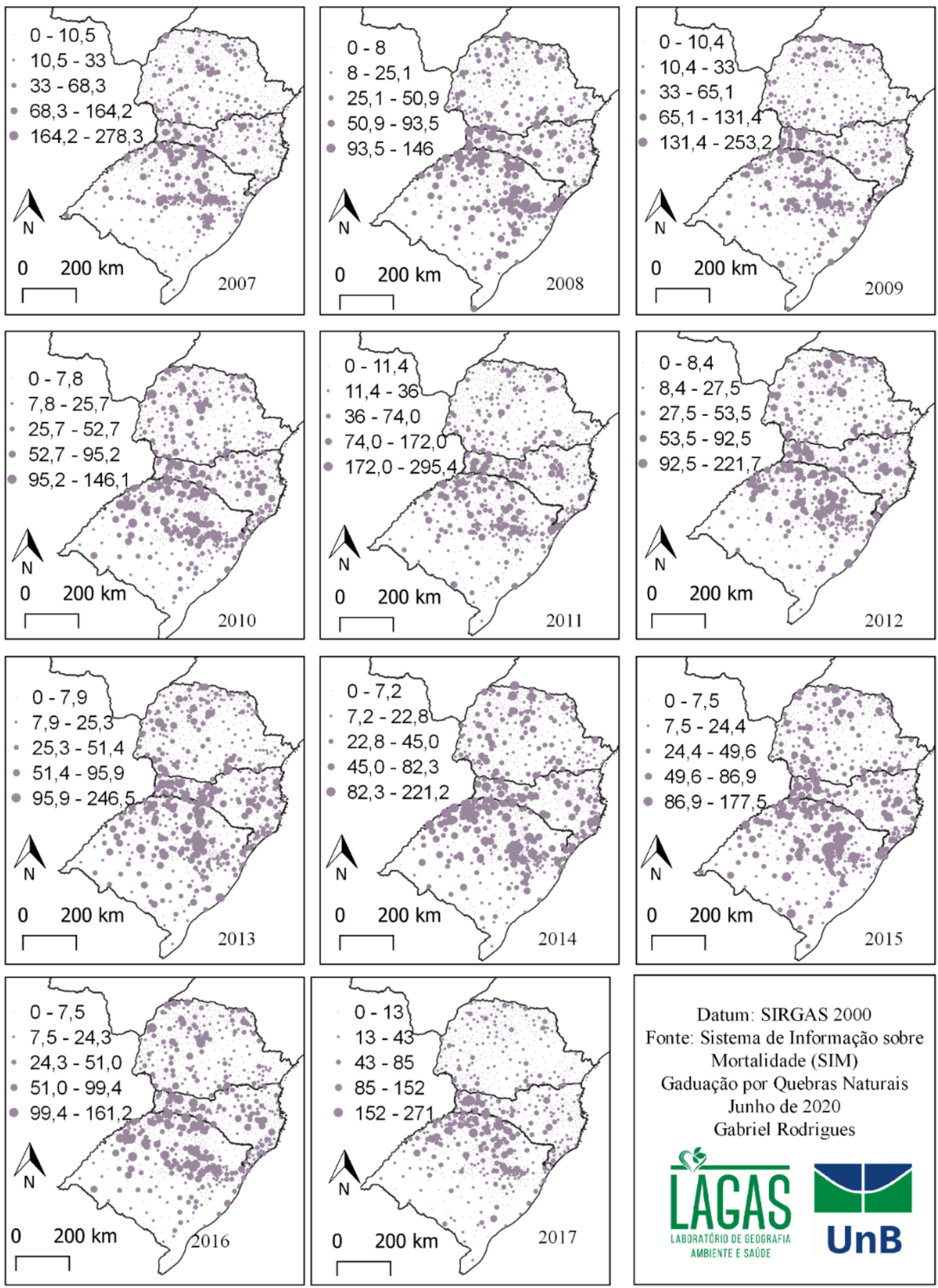

Fonte: MINISTÉRIO DA SAÚDE, 2020. Elaboração: os autores (2020). 
Oliveira, Silva, Teixeira, Malta e Iser (2021) explicam que a disparidade dentre os sexos não é completamente conhecida, mas a maior exposição ao álcool e tabaco podem ser fatores preponderantes. Os autores também trazem que houve uma queda na mortalidade feminina por esta enfermidade desde 1990 a 2017, possivelmente relacionada ao aumento da procura por serviços de saúde recentemente, sendo feito mais frequente por mulheres.

\section{Faixa etária}

A pirâmide etária dos falecidos pela causa base "câncer de esôfago" une os dados de sexo e faixa etária em uma representação gráfica (Figura 5). Em todos os estados analisados, os homens, além de serem maioria, também acumulam o pico de mortes nas faixas etárias mais baixas em comparação aos dados do sexo feminino. Resultados de Braga, Oliveira e Dutra (2011) apontaram para uma conclusão semelhante, pois a faixa etária de maior incidência do câncer de esôfago, no recorte estudado por eles, foi entre 55 a 60 anos, assim como Mota, Curado (et al, 2013) que identificaram um risco maior em pacientes acima de 55 anos.

Figura 5 - Pirâmide etária dos óbitos por C15 na Região Sul do Brasil no período de 2007 a 2017.

Pirâmide etária dos falecidos por câncer de esôfago na Região Sul (2007-2017)

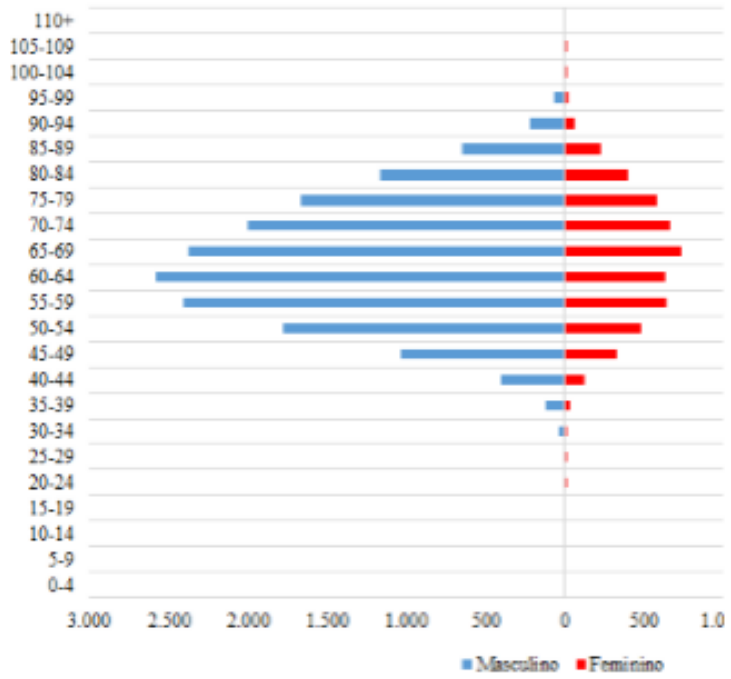

Pirâmide etária dos falecidos por câncer de esôfago no Paraná (2007-2017)

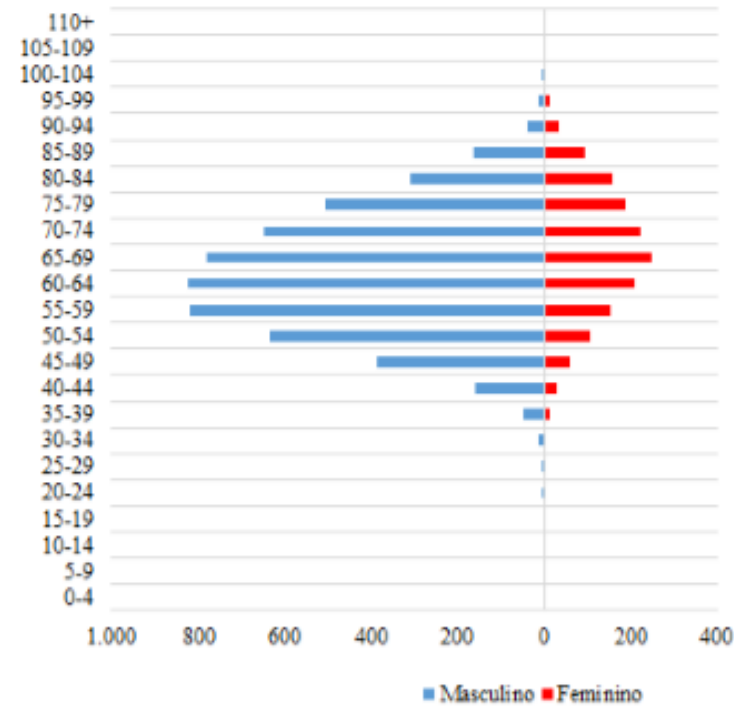

Pirâmide etária dos falecidos por câncer de esôfago no Rio Grande do Sul (2007-2017)

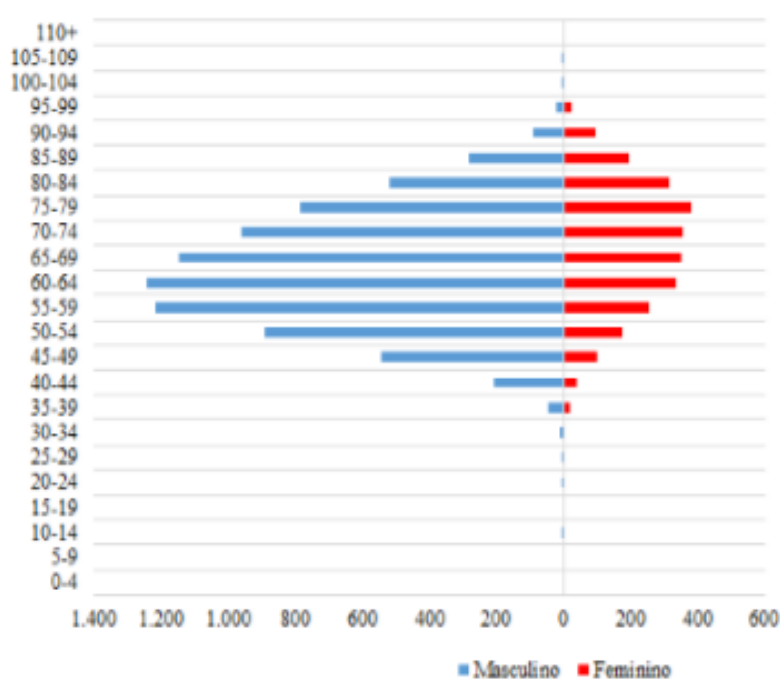

Pirâmide etária dos falecidos por câncer de esôfago em Santa Catarina (2007-2017)

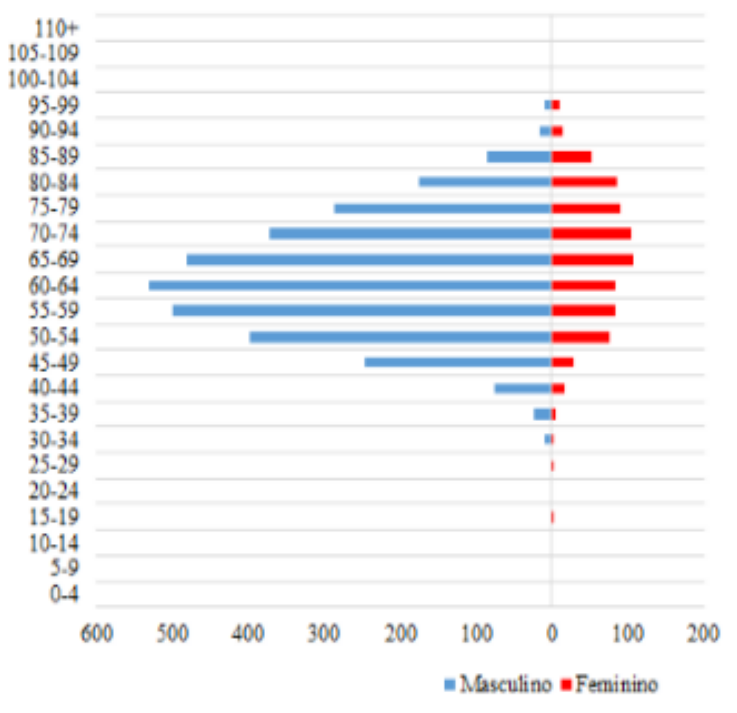

Fonte: MINISTÉRIO DA SAÚDE, 2020. Elaboração: os autores (2020).

Hygeia Uberlândia - MG $\quad$ Edição especial: X GeoSaude $\quad$ Fev./2022 p. 155-167 Página 163


Enquanto a maioria das mulheres falecem pelo câncer de esôfago no Rio Grande do Sul na faixa etária dos 75-79 anos, os homens do mesmo estado alcançam a marca aos 60-64 anos. No Paraná e em Santa Catarina, a diferença é reduzida, ainda assim, o pico de óbito de mulheres é na faixa dos 65-69 anos e o dos homens é aos 60-64 anos.

A maior concentração na população de idade avançada, conforme Oliveira (et al, 2021), pode estar associada a características cumulativas de exposição à fatores carcinogênicos, destacando a exposição à fumaça. No entanto, os autores apontam no estudo que a concentração da mortalidade pelo câncer de esôfago está na população com mais de 70 anos no Brasil, enquanto este estudo aponta para um acumulado de óbitos nos grupos acima dos 55 anos, sendo os grupos de 60 a 64 anos os maiores do Sul do Brasil.

A Pesquisa Nacional de Saúde aponta para uma possível explicação sobre a diferença de idade e sexo na mortalidade do câncer de esôfago. Segundo os dados de 2013, nos três estados do Sul as mulheres começam, na média, a consumir álcool e tabaco mais tarde na vida do que os homens (IBGE, 2013). Os homens também tem maior proporção de pessoas de 18 anos ou mais de idade que costumam consumir bebida alcoólica uma vez ou mais por semana nos três estados. No Paraná, $36 \%$ dos homens fazem ingestão de álcool, enquanto a proporção de mulheres no mesmo ano é de $16 \%$. Em Santa Catarina e no Rio Grande do Sul, respectivamente, $41,7 \%$ e 47,0\% dos homens consomem álcool uma vez ou mais na semana e as consumidoras são $15,2 \%$ e 17,3\% da população estadual feminina.

Sobre o consumo abusivo de álcool, a PNS relata que os homens são maioria no consumo excessivo do produto nos últimos trinta dias antes da pesquisa, sendo 16,5\% no Paraná, 17,3\% em Santa Cataria e 18,9\% no Rio Grande do Sul. Em comparação, no grupo de mulheres apenas 5,2\% delas relataram no Paraná o consumo excessivo durante o mesmo período, 5,7\% em Santa Catarina e 4,8\% no Rio Grande do Sul (IBGE, 2013).

Durante o inquérito da PNS, $21,6 \%$ dos homens com mais de 18 anos eram fumantes atuais de tabaco no Paraná e das mulheres na mesma faixa etária $14,9 \%$ consumiam o mesmo produto. Em Santa Catarina, $12,5 \%$ das mulheres fumavam durante o período da pesquisa, enquanto dos homens esse público era de 19,7\%. O Rio Grande do Sul tinha uma proporção menor de usuários, 16,4\% dos homens e $12,3 \%$ das mulheres (IBGE, 2013).

Como pode ser visto no quadro síntese (Quadro 2) e na análise exploratória (Figura 1), o Rio Grande do Sul tem as maiores taxas de mortalidade por câncer de esôfago no Sul, tem a maior proporção de consumidores de álcool de ambos os sexos e maior proporção de homens consumidores abusivos de álcool. Entretanto, tem as menores proporções de homens e mulheres fumantes, menor proporção de mulheres consumidoras abusivas de álcool da região e ápice de mortalidade de mulheres mais tardiamente na vida.

Quadro 2 - Quadro síntese dos óbitos e fatores de risco álcool e fumo.

\begin{tabular}{|c|c|c|c|c|}
\hline & Estados/ & $P R$ & SC & RS \\
\hline \multirow[b]{2}{*}{ Proporção dos óbitos por câncer de esôfago } & Homens & $77,7 \%$ & $80,8 \%$ & $75,0 \%$ \\
\hline & Mulheres & $22,3 \%$ & $19,2 \%$ & $25,0 \%$ \\
\hline \multirow[b]{2}{*}{ Proporção dos consumidores de álcool (em relação a população geral) } & Homens & $36,0 \%$ & $41,7 \%$ & $47,0 \%$ \\
\hline & Mulheres & $16,0 \%$ & $15,2 \%$ & $17,3 \%$ \\
\hline \multirow[b]{2}{*}{ Proporção dos consumidores abusivos de álcool (em relação a população ger } & Homens & $16,5 \%$ & $17,3 \%$ & $18,9 \%$ \\
\hline & Mulheres & $5,2 \%$ & $5,7 \%$ & $4,8 \%$ \\
\hline \multirow[b]{2}{*}{ Proporção dos fumantes (em relação a população geral) } & Homens & $21,6 \%$ & $19,7 \%$ & $16,4 \%$ \\
\hline & Mulheres & $14,9 \%$ & $12,5 \%$ & $12,3 \%$ \\
\hline \multirow[b]{2}{*}{ Faixa etária com mais óbitos } & Homens & $60-64$ & $60-64$ & $60-64$ \\
\hline & Mulheres & $65-69$ & 65-69 & $75-79$ \\
\hline
\end{tabular}

Fonte: IBGE, 2013 e MINISTÉRIO DA SAÚDE, 2020. Elaboração: os autores (2020).

O estado de Santa Catarina reveza, geralmente, as terceiras e as quartas maiores taxas de mortalidade de câncer de esôfago com o Paraná (Quadro 2). O estado catarinense tem o menor número absoluto de óbitos por esta enfermidade na região, mas tem a maior proporção de homens falecidos, maior proporção de homens consumidores abusivos de álcool, segunda maior proporção de homens consumidores de álcool e proporção de fumantes. As mulheres do estado lideram a proporção em consumo de álcool e falecem mais em grupo etário mais jovem que o Rio Grande do Sul.

Hygeia Uberlândia - MG $\quad$ Edição especial: X GeoSaude $\quad$ Fev./2022 $\quad$ p. 155-167 Página 164


O Paraná, assim como Santa Catarina, tem a faixa etária em que as mulheres mais morrem mais cedo que no Rio Grande do Sul, tendo também as maiores proporção de mulheres e homens fumantes da região (Quadro 2). Em relação ao álcool, as mulheres tem as segundas maiores proporções da região, da mesma forma que na proporção de sexo dos óbitos por câncer de esôfago.

Outro fator de risco digno de discussão neste artigo é a obesidade, explicitada dentre os principais fatores de risco nas Diretrizes Diagnósticas e Terapêuticas para o carcinoma esofágico do Brasil (BRASIL, 2014). Rezende e Melinda (et al, 2018) apresentam que 3,8\% dos novos casos de câncer diagnosticados no Brasil são atribuídos ao alto Índice de Massa Corporal (IMC). Segundo a projeção do mesmo estudo, em 2025 o alto IMC será responsável por 29.490 casos de câncer, sendo 18.837 casos em mulheres e 10.653 em homens. Segundo Aguiar e Souza (et al, 2019), o adenocarcinoma de esôfago corresponde a $4 \%$ dos cânceres da população com obesidade da América Latina.

O envelhecimento da população é de significativa relevância para a incidência do câncer de esôfago, uma vez que esse tipo de câncer é raro durante a juventude e apresenta alta incidência em idosos e sabe-se que a exposição prolongada a agentes cancerígenos no ambiente seja o principal fator de risco (GIMENO, et al., 1995). Logo, em uma população com maior número de idosos, como no estágio da transição demográfica para qual o Brasil está caminhando, deve haver um aumento no número de casos de câncer no esôfago e, possivelmente, uma sobrecarga nos centros de tratamento caso não haja um planejamento.

Sabendo que mesmo para os indivíduos mais cuidadosos e bem informados, nem todo risco é evitado e que ao mesmo tempo a redução de riscos é possível, recomenda-se o hábito de exercitar-se, uma boa nutrição seguindo as recomendações médicas capaz de evitar exposições regulares à agentes carcinogênicos ingeridos (MCKINNELL, 2008). Gallus, Bosetti et al. (2001), por exemplo, defendem o potencial do consumo de vegetais e frutas na prevenção ao câncer de esôfago. Pool-Zabel et al. (apud BAÚ; HUTH, 2011) vão além ao mencionar o benefício do consumo do carotenoide, presente no espinafre, cenoura e tomate.

\section{CONSIDERAÇÕES FINAIS}

Este estudo contribui para a temática ao atualizar o perfil de sexo e faixa etária da Região que, quando mencionada, não ultrapassava a análise regional, impossibilitando a comparação da análise da distribuição espacial das taxas de mortalidade por sexo com outros trabalhos.

O câncer de esôfago apresenta uma alta fatalidade devido ao seu diagnóstico frequentemente em estágio avançado. No entanto, conhecendo o seu perfil e as áreas de maior frequência é possível criar companhas de prevenção focadas em grupos mais afetados.

Compreendendo que o cuidado à saúde de forma integral é uma das sínteses dos sistemas públicos de saúdes universais, no qual se enquadra o Sistema Único de Saúde (SUS), destaca-se que a compreensão das populações de interesse para determinado agravo ou desfecho de saúde é importante, como já referenciado, para planejamento/estabelecimento de uma assistência em saúde de agravos de interesse (STARFIELD, 2002; BRASIL, 2010).

O presente estudo indica que para a Região Sul do Brasil os homens maiores de 60 anos constituem a população de interesse para as ações de atenção integral à saúde. Nesse sentido, pode ser considerado como uma contribuição efetiva para a elaboração de estratégias de base local com vistas à redução de morbimortalidade por câncer de esôfago.

Identificamos como limitação a organização do câncer de esôfago na CID-10 utilizada pelo SIM em oito variações além de C15 na coluna "CAUSABASE", nas quais não diferenciam entre os casos de carcinoma das células escamosas do esôfago (CEE) e o adenocarcinoma. Devido a organização da CID-10 se limitar à posição das células cancerosas não foi possível analisar as doenças separadamente, mesmo havendo diferenciações entre fatores de risco e possivelmente na distribuição espacial, afetando a efetividade de políticas públicas especializadas.

\section{AGRADECIMENTOS}

O presente trabalho foi realizado com apoio do Laboratório de Geografia Ambiente e Saúde da Universidade de Brasília (LAGAS/UnB), e dos projetos UnB/IRD JEAI-GITES e UnB/FIOCRUZ/IRD LMI-Sentinela.

\section{REFERÊNCIAS}

AGUIAR, M. et al. Perfil populacional da obesidade associada ao câncer na América Latina e no mundo.

Revista Saúde \& Ciência Online, v. 8, n. 2, p.125-133, 2019. https://doi.org/10.35572/RSC.V8I2.838.

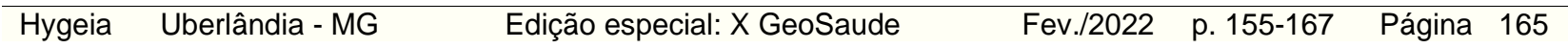


ASSMANN, N. S. ERVA-MATE: O alicerce de uma identidade histórica, cultural, econômica e espiritual. Trabalho de Conclusão de Curso (Tecnológico em Gastronomia) - Instituto Federal de Santa Catarina, Florianópolis, 2018.

BARROS, S. G. S.; et al. Mate (chimarrão) é consumido em alta temperatura por população sob risco para o carcinoma epidermóide de esôfago. Arquivos de Gastroenterologia (Impresso), São Paulo, v. 37, n.1, p. 25-30, 2000. https://doi.org/10.1590/S0004-28032000000100006.

BAÚ, F.; HUTH, A. Fatores de risco que contribuem para o desenvolvimento do câncer gástrico e de esôfago. Revista Contexto \& Saúde, ljuí, v. 11, n. 21, p 16-24, 2011. https://doi.org/10.21527/21767114.2011.21.16-24.

BRAGA, R.; DUTRA, D.; OLIVEIRA, M. Incidência Geopatológica de Neoplasia no Brasil. Revista Inspirar, v. 3, p. 58-62, 2011.

BRASIL. Ministério da Saúde. Portaria GM no 1.439, de 16 de dezembro de 2014. Aprova as Diretrizes Diagnósticas e Terapêuticas do Carcinoma de Esôfago. Diário Oficial da União, 16 dez. 2014.

, Ministério da Saúde. Secretaria de Atenção à Saúde. Departamento de Atenção Básica.

Rastreamento / Ministério da Saúde, Secretaria de Atenção à Saúde, Departamento de Atenção Básica. Brasília: Ministério da Saúde, 2010. 95 p.: il. - (Série A. Normas e Manuais Técnicos) (Cadernos de Atenção Primária, n. 29). ISBN 978-85-334-1729-8.

CARMO, E. Emergências de saúde pública: breve histórico, conceitos e aplicações. Saúde em Debate, v. 44, p. 9-19, 2020. https://doi.org/10.1590/0103-11042020e201.

CASTELLSAGUÉ, X.; et al. Influence of mate drinking, hot beverages and diet on esophageal cancer risk in South America. International Journal of Cancer, v. 88, n. 4, p. 658-664, 2000.

https://doi.org/10.1002/1097-0215(20001115)88:4\%3C658::AID-IJC22\%3E3.0.CO;2-T

GALLUS S.; et al. Oesophageal cancer in women: Tobacco, alcohol, nutritional and hormonal factors.

British Journal of Cancer, v. 85, n. 3, p.341-345, 2001. https://doi.org/10.1054/bjoc.2001.1898

GIMENO, S.; et al. Fatores de risco para o câncer de esôfago: estudo caso-controle em área metropolitana da região Sudeste do Brasil. Revista de Saúde Pública, São Paulo, v. 29, n.3, p. 159-165, 1995. https://doi.org/10.1590/S0034-89101995000300002.

GUERRA, M.; GALLO, C.; MENDONÇA, G. Risco de câncer no Brasil: tendências e estudos epidemiológicos mais recentes. Revista Brasileira de Cancerologia, Rio de Janeiro, v. 51, n. 3, p. 227234, 2005. https://doi.org/10.32635/2176-9745.RBC.2005v51n3.1950.

INCA - Instituto Nacional do Câncer. Estatísticas de câncer. Nacional Cancer Institute. Disponível em: https://www.inca.gov.br/en/node/2244. Acesso em: 01 de abr. 2020.

ISLAMI, F.; et al. Tea drinking habits and oesophageal cancer in a high-risk area in northern Iran: population based case-control study. BMJ [online], p. 1-8, 2009. https://doi.org/10.1136/bmi.b929.

JONG, U.; et al. Aetiological factors in oesophageal cancer in Singapore Chinese. International Journal of Cancer, [S. I.], 1974. https://doi.org/10.1002/ijc.2910130304.

KOCA, T.; et al. Dietary and Demographical Risk Factors for Oesophageal Squamous Cell Carcinoma in the Eastern Anatolian Region of Turkey Where Upper Gastrointestinal Cancers are Endemic. Asian Pacific Journal of Cancer Prevention, v. 16, 2015. https://doi.org/10.7314/APJCP.2015.16.5.1913.

LAUNOY, G.; et al. Oesophageal cancer in France: Potential importance of hot alcoholic drinks. International Journal of Cancer, v. 71, n. 6, p.917-923, 1998. https://doi.org/10.1002/(SICl)10970215(19970611)71:6<917::AID-IJC1>3.0.CO;2-0.

LIN, J.; et al. Hot Beverage and Food Intake and Esophageal Cancer in Southern China. Asian Pacific Journal of Cancer Prevention, v. 12, n. 9, p. 2189-2192, 2011.

MCKINNELL, R. Prevention of Cancer. 1.ed. Nova York: Chelsea House, 2008.

MINISTÉRIO DA SAÚDE, 2020. DATASUS. Sistema de Informações de Mortalidade - SIM

MOTA, O. M. da; CURADO, M. P.; OLIVEIRA, J. C.; CARDOSO, D. M. M.; MARTINS, E. Risk factors for esophageal cancer in a low-incidence area of Brazil. São Paulo Medical Journal (Impresso), v. 131, p. 27-34, 2013. https://doi.org/10.1590/S1516-31802013000100005.

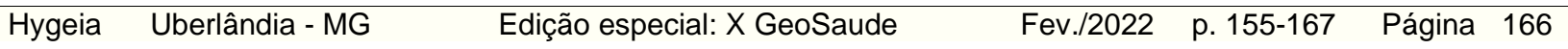


MUNISH, M.; et al. Africa's oesophageal cancer corridor: Do hot beverages contribute?. Cancer Case Control, v. 26, n. 10, p. 1477-1487, 2015. https://doi.org/10.1007/s10552-015-0646-9.

MUÑOZ, N.; et al. PRECURSOR LESIONS OF OESOPHAGEAL CANCER IN HIGH-RISK POPULATIONS IN IRAN AND CHINA. The Lancet, [S. I.], 1982. https://doi.org/10.1016/S01406736(82)92151-1.

OLIVEIRA, M.; et al. ESOPHAGEAL CANCER MORTALITY IN BRAZIL: A TIME-SERIES ANALYSIS FROM THE GLOBAL BURDEN OF DISEASE STUDY. Arquivos de Gastoenterologia (ONLINE), v. 58, p. 100-106, 2021. https://doi.org/10.1590/s0004-2803.202100000-17.

OLIVEIRA, Y. M. M. de; ROTTA, E. Área de distribuição natural de erva-mate (llex paraguariensis St. Hil). In: SEMINÁRIO SOBRE ATUALIDADES E PERSPECTIVAS FLORESTAIS - SILVICULTURA DA ERVAMATE, 1983, Curitiba. Anais... Curitiba, EMBRAPA/CNPF, 1983. p. 17-35. Disponível em: https://www.alice.cnptia.embrapa.br/alice/handle/doc/300244. Acesso em: 19 out. 2020.

IBGE - INSTITUTO BRASILEIRO DE GEOGRAFIA E ESTATíSTICA. Pesquisa Nacional de Saúde do Escolar 2013. Rio de Janeiro: IBGE; 2013. Disponível em: http://www.pns.fiocruz.br. Acesso em 20 out. 2020.

PROLLA, J. C.; DIETZ, J.; COSTA, L. A. Diferenças geográficas na mortalidade por câncer de esôfago no Rio Grande do Sul. Revista da Associação Médica Brasileira, v. 39, p. 217-220, 1993.

REZENDE, L.; et al. The increasing burden of cancer attributable to high body mass index in Brazil. Journal Cancer Epidemiology, v. 54. p. 63-70, jun. 2018. https://doi.org/10.1016/..canep.2018.03.006. STEFANI, E.; et al. Mate Drinking, Alcohol, Tobacco, Diet, and Esophageal Cancer in Uruguay. Cancer Research, v. 50, n. 2, p. 426-431, 1990.

STARFIELD, B. Atenção primária: equilíbrio entre necessidades de saúde, serviços e tecnologia. Brasília: UNESCO, Ministério da Saúde, 2002. 726p. ISBN:85-87853-72-4

VICTORA, C. G.; et al. Hot beverages and oesophageal cancer in southern Brazil: A case-control study. International Journal of Cancer, v. 39, n.6, p. 710-716, 1987. https://doi.org/10.1002/ijc.2910390610.

WHO - WORLD HEALTH ORGANIZATION. International Agency for Research on Cancer - IARC. World Cancer Report. Lyon: WHO; 2014.

WORLD HEALTH ORGANIZATION. List of Classifications by cancer sites with sufficient or limited evidence in humans. v. 1-125, 2019.

WHO - WORLD HEALTH ORGANIZATION. International Agency for Research on Cancer - IARC. World Cancer Report. Lyon: WHO; 2020.

WORLD HEALTH ORGANIZATION. International Agency for Research on Cancer - IARC. PRESS RELEASE N² 244. Lyon: WHO; 2016. 